\title{
Optical Conductivity of Ultra-Cold Bosons in Optical Lattices
}

\author{
B. Grygiel, K. Patucha And T.A. Zaleski \\ Institute of Low Temperature and Structure Research, Polish Academy of Sciences, \\ P.O. Box 1410, 50-950 Wrocław, Poland
}

\begin{abstract}
We study ultra-cold bosonic systems in optical lattice using quantum rotor approach to calculate the currentcurrent correlations, which provides the information about conductivity of the system. The method allows us to go beyond mean-field approximation and track the behavior of the real part of the conductivity along the phase transition between the Mott insulator and superfluid state for various geometries of the lattice. In the phaseordered state at zero temperature, a discrete ingredient appears resulting from the long-range coherence in the system.
\end{abstract}

DOI: 10.12693/APhysPolA.130.633

PACS/topics: $67.85 . \mathrm{Hj}$, 05.30.Jp, 03.75.Lm

\section{Introduction}

Transport properties of strongly interacting systems are a focal point of research since discovery of the superconductivity and quantum Hall effect [1, 2]. The Fermi liquid theory, although it is sufficient to describe electronic transport in simple metals, does not take into account the presence of the lattice. Thus, different approach is needed to accommodate periodic systems with strong interactions between electrons.

Thanks to recent development in the cooling and trapping techniques an excellent testing ground for the solid state physics has arisen in the form of optical lattice filled with ultra-cold atoms $[3,4]$. These systems are created by the interference of counter-propagating laser beams. The periodic pattern of light intensity traps ultra-cold atoms due to ac Stark shift, creating a model of a system of particles moving in a tight-binding scheme in a lattice (e.g. electrons in strongly-correlated solids). Parameters of such systems, like tunnelling strength, interaction energy, dimensionality and geometry, are easily controlled in wide range. When the tunnelling overcomes the repulsive interaction between atoms, the system becomes a superfluid (SF), characterized by a long-range phase coherence. As the interaction energy increases, the atoms tend to localize on the lattice sites, creating incompressible Mott insulator (MI). Although, number of probes to investigate the state of ultra-cold atoms is limited in comparison to solid-state physics, recently an experiment of Krinner et al. [5] has shown a method of measuring optical conductance in an optical lattice version of quantum point contact. The flow between two reservoirs of ultra-cold atoms was triggered by the off-set in chemical potential between them. The quantized conductance was derived based on the measurement of the relative particle number imbalance. This experiment motivates our study of transport properties in neutral bosonic systems.

The paper is constructed in the following way. The first two sections contain an introduction - a short description of quantum rotor approach to the Bose-Hubbard model and a derivation of the formula for optical conductivity. Section 4 contains frequency dependence of optical conductivity along with a brief analysis of its dependence on system parameters and presence of the superfluid phase. In the last section, the results are summarized.

\section{Model}

To describe a system of strongly interacting bosonic particles in optical lattice, the Bose-Hubbard (BH) model is employed $[6,7]$. Its Hamiltonian has the following form:

$$
\begin{aligned}
\hat{H} & =-\sum_{\left\langle\boldsymbol{r}, \boldsymbol{r}^{\prime}\right\rangle} t_{\boldsymbol{r}, \boldsymbol{r}^{\prime}}\left[\hat{a}^{\dagger}(\boldsymbol{r}) \hat{a}\left(\boldsymbol{r}^{\prime}\right)+\text { H.c. }\right] \\
& +\frac{U}{2} \sum_{\boldsymbol{r}} \hat{n}^{2}(\boldsymbol{r})-\bar{\mu} \sum_{\boldsymbol{r}} \hat{n}(\boldsymbol{r}),
\end{aligned}
$$

where $\hat{a}(\boldsymbol{r})\left(\hat{a}^{\dagger}(\boldsymbol{r})\right)$ are bosonic annihilation (creation) operators and $\hat{n}(\boldsymbol{r})$ is the number operator at the lattice site $\boldsymbol{r}$. The first term describes hopping between the nearest neighbours with matrix element $t_{\boldsymbol{r}, \boldsymbol{r}^{\prime}}$, while the second - on-site repulsive interaction between particles. The last term contains shifted chemical potential, $\bar{\mu}=\mu+U / 2$ and serves as a tool for controlling the number of particles.

The exact derivation of the quantum rotor approach can be found in [8-11]. Here we present the general idea behind the method and the final outcome. We start writing the partition function in the path integral formalism

$$
Z=\int[\mathcal{D} \bar{a} \mathcal{D} a] \mathrm{e}^{-S[\bar{a}, a]},
$$

with the action of the form

$$
\begin{gathered}
S[\bar{a}, a]=\int_{0}^{\beta} \mathrm{d} \tau H[\bar{a}(\tau), a(\tau)] \\
+\int_{0}^{\beta} \mathrm{d} \tau \sum_{\boldsymbol{r}} \bar{a}(\boldsymbol{r}, \tau) \frac{\partial}{\partial \tau} a(\boldsymbol{r}, \tau),
\end{gathered}
$$

where the $0 \leq \tau \leq \beta$ is the imaginary time and $\beta \equiv$ $1 / k_{\mathrm{B}} T$ with $T$ being temperature.

The crucial element of the quantum rotor approach is a gauge transformation of the complex fields $a$ and $\bar{a}$ :

$$
a(\boldsymbol{r}, \tau)=\mathrm{e}^{\mathrm{i} \phi(\boldsymbol{r}, \tau)} b(\boldsymbol{r}, \tau),
$$




$$
\bar{a}(\boldsymbol{r}, \tau)=\mathrm{e}^{-\mathrm{i} \phi(\boldsymbol{r}, \tau)} \bar{b}(\boldsymbol{r}, \tau),
$$

which introduces new complex amplitude $b$ and phase $\phi$ fields with $b$ controlling the superfluid density and $\phi-$ long-range phase coherence. This leads to a separation of phase and amplitude degrees of freedom. Since the phase transition between superfluid and the Mott insulator phases is governed by phase ordering, amplitude fluctuations can be omitted leading to an average meanfield value of the amplitude $b_{0}=\sqrt{(t z+\bar{\mu}) / U}$, where $z$ is the coordination number of the lattice. As a result, the action depends only on the phase variables and takes form of the action of interacting quantum rotors

$$
\begin{aligned}
& S[\phi]=\int_{0}^{\beta} \mathrm{d} \tau\left\{-2 J \sum_{\left\langle\boldsymbol{r}, \boldsymbol{r}^{\prime}\right\rangle} \cos \left(\phi\left(\boldsymbol{r}^{\prime}, \tau\right)-\phi(\boldsymbol{r}, \tau)\right)\right. \\
& \left.+\sum_{\boldsymbol{r}}\left[\frac{\dot{\phi}^{2}(\boldsymbol{r}, \tau)}{2 U}+\mathrm{i} \frac{\bar{\mu}}{U} \dot{\phi}(\boldsymbol{r}, \tau)\right]\right\},
\end{aligned}
$$

where $J=t(t z+\bar{\mu}) / U$ is an effective coupling constant. Following the derivation presented in [8-10], one obtains the equation of state in the zero-temperature limit, which takes the following form:

$$
\begin{aligned}
& 1-\Psi^{2}= \\
& \frac{1}{2} \int \frac{\rho(E) \mathrm{d} E}{\sqrt{J(\varepsilon(0)-E) / U+\delta \lambda / U+v^{2}(\mu / U)}},
\end{aligned}
$$

where $\Psi^{2}$ denotes the order parameter (non-zero in the SF phase, zero in the MI phase and on the critical line). Parameter $\delta \lambda$ measures the distance from the critical point and vanishes in the SF state. The function $\rho(E)$ is the lattice density of states, $\varepsilon(\boldsymbol{k})$ is dispersion relation of a lattice and $v(x)=x-[x]-1 / 2$ with $[x]$ denoting the floor function. Setting $\Psi^{2}=0$ and $\delta \lambda=0$ the equation for the critical line is obtained from (6). The calculated phase diagrams are presented in the upper parts of Figs. 1-3.

Quantum rotor approximation to the Bose-Hubbard model has a great advantage of taking into account the spatial correlation in the system (included via the density of states function), thus allowing for analysis of its properties [12] for different types of lattices.

\section{Optical conductivity}

To study the transport properties of the system of ultra-cold bosons in optical lattice, the complex hopping term - Peierls factor - needs to be included in the $\mathrm{BH}$ Hamiltonian [13]:

$$
t \rightarrow t \exp \left(-\frac{2 \pi \mathrm{i}}{\Phi_{0}} \int_{\boldsymbol{r}}^{\boldsymbol{r}^{\prime}} \boldsymbol{A}(\boldsymbol{r}, \tau) \cdot \mathrm{d} \boldsymbol{l}\right),
$$

where $\boldsymbol{A}(\boldsymbol{r}, \tau)$ is a vector potential, here serving as a probe field, and $\Phi_{0}$ denotes the magnetic flux quantum. The optical conductivity can be expressed in terms of derivatives over the vector potential $\boldsymbol{A}$ [14-17]:

$$
\sigma_{i j}\left(\kappa, \omega_{\nu}\right)=\frac{1}{N \beta \omega_{\nu}}
$$

$$
\begin{aligned}
& \times\left.\sum_{\boldsymbol{r}, \boldsymbol{r}^{\prime}} \int_{0}^{\beta} \mathrm{d} \tau \mathrm{d} \tau^{\prime} \frac{\delta^{2} \ln Z}{\delta A_{i}(\boldsymbol{r}, \tau) \delta A_{j}\left(\boldsymbol{r}^{\prime}, \tau^{\prime}\right)}\right|_{\boldsymbol{A}=0} \\
& \times \exp \left(-\mathrm{i} \omega_{\nu}\left(\tau-\tau^{\prime}\right)\right) \exp \left(-\mathrm{i} \kappa\left(\boldsymbol{r}-\boldsymbol{r}^{\prime}\right)\right),
\end{aligned}
$$

where $\boldsymbol{A}$ is assumed to be independent of the position $\boldsymbol{r}$ The action (3) can be rewritten as a sum $S=S_{0}+S^{\prime}$, where

$$
\begin{aligned}
S_{0} & =-\int_{0}^{\beta} \mathrm{d} \tau \\
& \times \sum_{\left\langle\boldsymbol{r}, \boldsymbol{r}^{\prime}\right\rangle}\left[t \mathrm{e}^{-\frac{2 \pi \mathrm{i}}{\Phi_{0}} \int_{\boldsymbol{r}}^{\boldsymbol{r}^{\prime}} \boldsymbol{A} \cdot \mathrm{d} \boldsymbol{l}} \bar{a}\left(\boldsymbol{r}^{\prime}, \tau\right) a(\boldsymbol{r}, \tau)+\text { c.c. }\right]
\end{aligned}
$$

and $S^{\prime}$ contains the remaining terms.
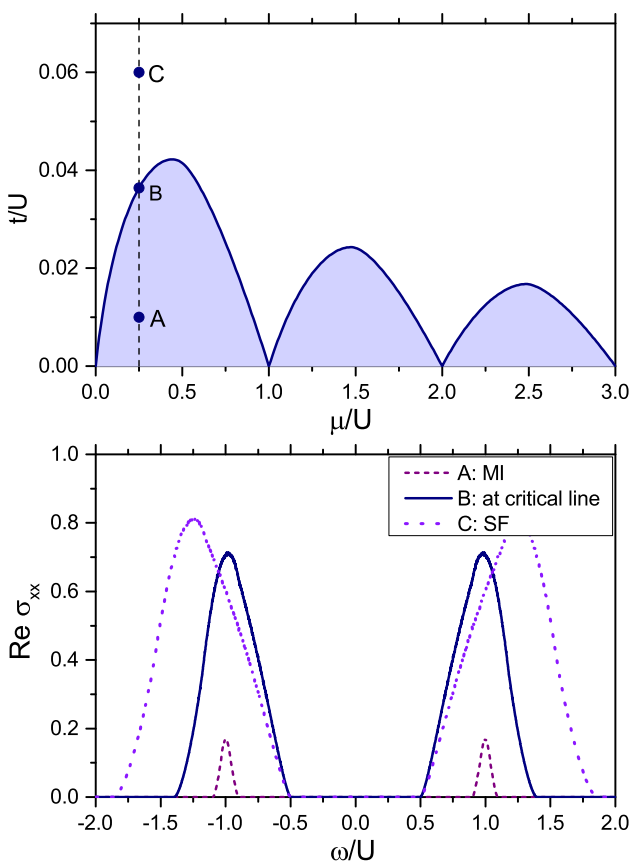

Fig. 1. Top: Phase diagram of SF-MI phase transition for simple cubic lattice. For the three points A, B, and $\mathrm{C}$ on the line $\mu=0.25$ the regular part of optical conductivity was drawn. Point A belongs to the Mott insulator phase, point B lies exactly at the critical line, and point $\mathrm{C}$ is located in the superfluid region. Bottom: The dependence of regular part of optical conductivity on frequency for simple cubic lattice for $\mu=0.25$. Lines A, B, C correspond to points A, B, C on the top graph.

Performing spatial Fourier transform of the action (9) yields in a general case of a system with multiple bands

$$
\begin{aligned}
S_{0} & =-\frac{t}{N} \sum_{\boldsymbol{k}, b} \int_{0}^{\beta} \mathrm{d} \tau \varepsilon_{b}\left(\boldsymbol{k}+\frac{2 \pi}{\Phi_{0}} \boldsymbol{A}(\tau)\right) \\
& \times \bar{a}_{\boldsymbol{k}}^{b}(\tau) a_{\boldsymbol{k}}^{b}(\tau),
\end{aligned}
$$

where $b=1, \ldots, q$ enumerates the band and $\varepsilon_{b}(\boldsymbol{k})$ denotes the dispersion relation of each band. Since wavevector $\boldsymbol{k}$ is shifted by the vector potential $\boldsymbol{A}$, the functional derivative over $\boldsymbol{A}$ can be effectively changed into derivative over $\boldsymbol{k}$ : 


$$
\begin{aligned}
& \frac{\delta \varepsilon_{b}\left(\boldsymbol{k}+\frac{2 \pi}{\Phi_{0}} \boldsymbol{A}\left(\tau^{\prime}\right)\right)}{\delta A_{i}(\tau)}= \\
& \frac{2 \pi}{\Phi_{0}} \delta\left(\tau-\tau^{\prime}\right) \frac{\partial \varepsilon_{b}\left(\boldsymbol{k}+\frac{2 \pi}{\Phi_{0}} \boldsymbol{A}\left(\tau^{\prime}\right)\right)}{\partial k_{i}} .
\end{aligned}
$$

After performing the time Fourier transform, the optical conductivity reads

$$
\begin{aligned}
& \sigma_{i j}\left(\omega_{\nu}\right)=\frac{1}{\omega_{\nu}} \frac{4 \pi^{2} t^{2}}{\Phi_{0}^{2}} \frac{1}{N^{3} \beta^{3}} \sum_{\boldsymbol{k}, b, m} \frac{\partial \varepsilon_{b}(\boldsymbol{k})}{\partial k_{i}} \frac{\partial \varepsilon_{b}(\boldsymbol{k})}{\partial k_{j}} \times \\
& {\left[\left\langle\bar{a}_{\boldsymbol{k}}^{b}\left(\omega_{m}+\omega_{\nu}\right) a_{\boldsymbol{k}}^{b}\left(\omega_{m}+\omega_{\nu}\right)\right\rangle\left\langle\bar{a}_{\boldsymbol{k}}^{b}\left(\omega_{m}\right) a_{\boldsymbol{k}}^{b}\left(\omega_{m}\right)\right\rangle+\right.} \\
& \left.-\left\langle\bar{a}_{\boldsymbol{k}}^{b}\left(\omega_{m}+\omega_{\nu}\right) a_{\boldsymbol{k}}^{b}\left(\omega_{m}+\omega_{\nu}\right)\right\rangle^{2}\right] .
\end{aligned}
$$
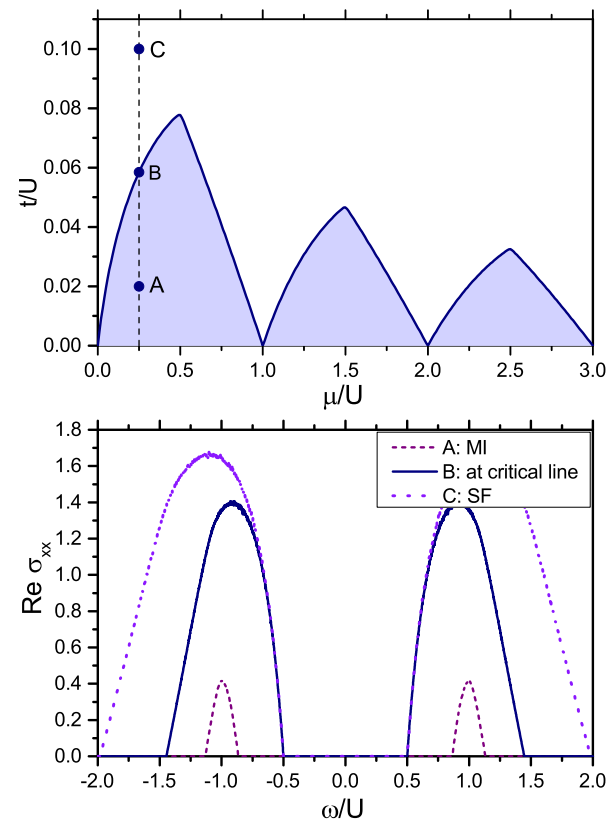

Fig. 2. Top: Phase diagram of SF-MI phase transition for square lattice. Bottom: The dependence of regular part of optical conductivity on frequency for square lattice $\mu=0.25$. Symbols are used in the same way as in Fig. 1.

The averages $\left\langle\bar{a}_{\boldsymbol{k}}^{b}\left(\omega_{m}\right) a_{\boldsymbol{k}}^{b}\left(\omega_{m}\right)\right\rangle$ calculated within the quantum rotor approach have the following form [11]:

$$
\begin{aligned}
& \left\langle\bar{a}_{\boldsymbol{k}}^{b}\left(\omega_{m}\right) a_{\boldsymbol{k}}^{b}\left(\omega_{m}\right)\right\rangle= \\
& \frac{N \beta b_{0}^{2}}{U\left\{\frac{J\left[\varepsilon_{b}(0)-\varepsilon_{b}(\boldsymbol{k})\right]}{U}+\frac{\delta \lambda}{U}+\frac{\omega_{m}^{2}}{U^{2}}-2 \mathrm{i} v\left(\frac{\mu}{U}\right) \frac{\omega_{m}}{U}\right\}} .
\end{aligned}
$$

The next step requires performing summation over the Matsubara frequencies $\omega_{m}=2 \pi m / \beta$ and analytical continuation to real frequencies $\mathrm{i} \omega_{m} \rightarrow \omega+\mathrm{i} 0^{+}$[8]. This leads to the final formula for the optical conductivity, which splits into singular and regular parts. The singular part is proportional to $\delta(\omega)$ :

$$
\operatorname{Re} \sigma_{\text {sing }}^{i j}(\omega)=\left.\frac{\pi^{3}}{4 \Phi_{0}^{2}} \frac{J^{2}}{U} \delta\left(\frac{\omega}{U}\right) \mathcal{A}(\beta, \delta \lambda)\right|_{\beta \rightarrow \infty}
$$
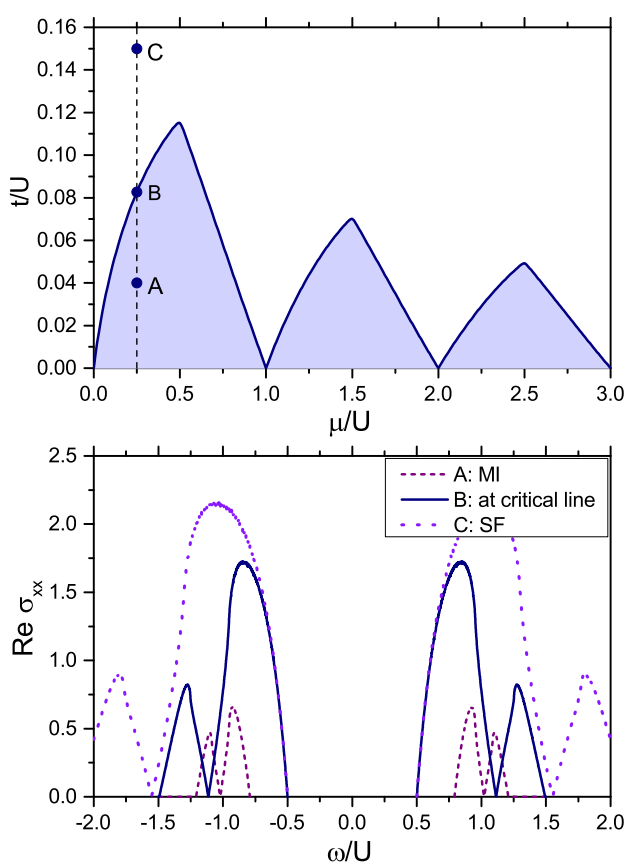

Fig. 3. Top: Phase diagram of SF-MI phase transition for honeycomb lattice. Bottom: The dependence of regular part of optical conductivity on frequency for honeycomb lattice $\mu=0.25$. Symbols are used in the same way as in Figs. 1 and 2.

with $\mathcal{A}(\beta, \delta \lambda)$ defined as:

$$
\begin{gathered}
\mathcal{A}(\beta, \delta \lambda)=\int \mathrm{d} E \frac{\beta \rho_{\mathrm{gen}}^{i j}(E)}{B^{2}} \\
\times\left\{\operatorname{csch}^{2}\left(\frac{U \beta}{2}\left[B-v\left(\frac{\mu}{U}\right)\right]\right)\right. \\
\left.+\operatorname{csch}^{2}\left(\frac{U \beta}{2}\left[B+v\left(\frac{\mu}{U}\right)\right]\right)\right\},
\end{gathered}
$$

where $B^{2}=J\left(E_{0}-E\right) / U+v^{2}(\mu / U)+\delta \lambda / U$. The behavior of this term is determined by the value of the Lagrange multiplier $\delta \lambda$. If $\delta \lambda=0$ (superfluid state), the term is non-zero and proportional to $\delta(\omega / U)$. Meanwhile, in the Mott insulator while $\delta \lambda \neq 0$ it disappears (actually it is still present in non-zero temperature, however it results from lack of dissipation in the system and is not connected with superfluid flow) $[8,14,18]$. The regular part, $\operatorname{Re} \sigma^{i j}(\omega)$, contains the current-current correlations

$$
\begin{aligned}
& \operatorname{Re} \sigma_{\text {reg }}^{i j}(\omega)= \\
& \frac{2 \pi^{3}}{\Phi_{0}^{2}} \frac{J}{U} \frac{\rho_{\text {gen }}^{i j}\left(\varepsilon_{q}(0)+\frac{\delta \lambda / U}{J / U}+\frac{v^{2}(\mu / U)}{J / U}-\frac{(\omega / U)^{2}}{4 J / U}\right)}{(\omega / U)^{2}}
\end{aligned}
$$

where $\rho_{\text {gen }}^{i j}(E)$ is generalized density of states defined as:

$$
\begin{aligned}
& \rho_{\text {gen }}^{i j}(E)=\frac{1}{q} \sum_{b=1}^{q} \frac{1}{V} \int_{\mathrm{BZ}} \mathrm{d} \boldsymbol{k} \delta\left(E-\varepsilon_{b}(\boldsymbol{k})\right) \\
& \times \frac{\partial \varepsilon_{b}(\boldsymbol{k})}{\partial k_{i}} \frac{\partial \varepsilon_{b}(\boldsymbol{k})}{\partial k_{j}} .
\end{aligned}
$$




\section{Results}

The dependence of the real, regular, longitudinal optical conductivity $\operatorname{Re} \sigma_{\text {reg }}^{x x}$ (denoted on the graphs as $\operatorname{Re} \sigma_{x x}$ ) on the frequency of the probing field is shown along with phase diagrams for three types of lattices simple cubic, square, and honeycomb in Figs. 1-3, respectively. As expected, the broadening of the peak of conductivity is observed as the kinetic term prevails and the system goes into SF state. For $\mu / U=0.25$, the energy gap $\omega_{g}$ equals $U$ at the critical line and in SF phase. In the Mott insulator phase the energy gap is larger than in the superfluid phase - this is due to a non-zero value of $\delta \lambda$ parameter (see Eq. (18)). One can observe that the optical conductivity of honeycomb lattice vanishes at certain frequency. This feature is strongly related to the existence of the Dirac cones, which causes the density of states to be 0 in the middle of the band [17]. The dependence of the energy gap on the distance from the critical line in the MI phase, described by the correction to the Lagrange multiplier $\delta \lambda$, can be derived from the condition of zeroing of conductivity. The dependence is given by the following formula:

$$
\omega_{g}=4 \sqrt{\delta \lambda+v^{2}\left(\frac{\mu}{U}\right)} .
$$

As the value of $\delta \lambda$ grows with decreasing hopping, the energy gap becomes larger. The relation (18) can be also applied to the system in the SF state, where $\delta \lambda$ is by definition equal to 0 . This indicates that the value of the energy gap is constant for $t \geq t_{c}$ (where $t_{c}$ is value of the hopping at the critical line) and a given chemical potential. An interesting feature can be observed for half-integer values of chemical potential (integer number of particles per lattice site) for the square lattice. In the superfluid state the energy gap closes (see Fig. 4) for $\mu / U=0.5$ (as can be easily deduced from Eq. (18)). This feature is in agreement with other results [14].

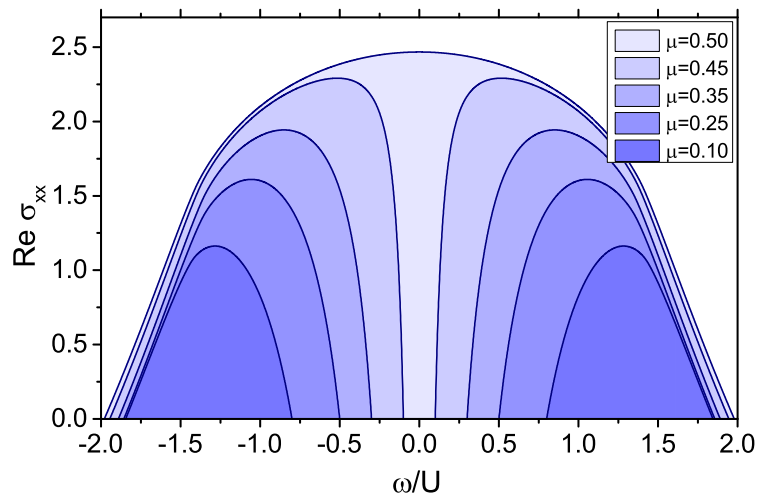

Fig. 4. The frequency dependence of the real, regular part of optical conductivity for a few values of chemical potential and constant hopping $t / U=0.1$ (in SF phase) for square lattice.

\section{Summary}

The transport properties of ultra-cold bosons in optical lattice has been studied using the Bose-Hubbard model. The Peierls substitution allowed for the derivation of the formula for the optical conductivity and thus, for the determination of the current-current correlations in the system. The Bose-Hubbard model was studied in the quantum rotor approach, which takes into account spatial correlations in the system. The real, regular part of the optical conductivity, which is connected to the current-current correlation function, was calculated for three lattice types — a simple cubic, a square, and a honeycomb lattices. As the quantum rotor approach does not lose the $U(1)$ symmetry of the Bose-Hubbard Hamiltonian, it provides the description of the behavior of the conductivity along the phase transition in the system of strongly-correlated bosons.

\section{References}

[1] J. Bardeen, L.N. Cooper, J.R. Schrieffer, Phys. Rev. 108, 1175 (1957).

[2] K. von Klitzing, G. Dorda, M. Pepper, Phys. Rev. Lett. 45, 494 (1980).

[3] I. Bloch, J. Dalibard, W. Zwerger, Rev. Mod. Phys. 80, 885 (2008).

[4] C. Pethick, H. Smith, Bose-Einstein Condensation in Dilute Gases, Cambridge University Press, 2002.

[5] S. Krinner, D. Stadler, D. Husmann, J.-P. Brantut, T. Esslinger, Nature 517, 64 (2015).

[6] D. Jaksch, C. Bruder, J.I. Cirac, C.W. Gardiner, P. Zoller, Phys. Rev. Lett. 81, 3108 (1998).

[7] M.P.A. Fisher, P.B. Weichman, G. Grinstein, D.S. Fisher, Phys. Rev. B 40, 546 (1989).

[8] T.K. Kopeć, J.V. José, Phys. Rev. B 60, 7473 (1999).

[9] T.K. Kopeć, Phys. Rev. B 70, 054518 (2004).

[10] T.P. Polak, T.K. Kopeć, Phys. Rev. B 76, 094503 (2007).

[11] T. Zaleski, T. Kopeć, Phys. Rev. A 84, 053613 (2011).

[12] T.A. Zaleski, Phys. Rev. A 85, 043611 (2012).

[13] D.R. Hofstadter, Phys. Rev. B 14, 2239 (1976).

[14] A.P. Kampf, G.T. Zimanyi, Phys. Rev. B 47, 279 (1993).

[15] M.-C. Cha, M.P.A. Fisher, S.M. Girvin, M. Wallin, A.P. Young, Phys. Rev. B 44, 6883 (1991).

[16] J. Wu, P. Phillips, Phys. Rev. B 73, 214507 (2006).

[17] A.S. Sajna, T.P. Polak, R. Micnas, Phys. Rev. A 89, 023631 (2014).

[18] A. van Otterlo, K.H. Wagenblast, R. Fazio, G. Schön, Phys. Rev. B 48, 3316 (1993). 